\title{
Effectiveness of a Walking Football Program for Middle-Aged and Older Men With Type 2 Diabetes: Protocol for a Randomized Controlled Trial
}

Ana Barbosa $^{1}, \mathrm{MSc}$ João Brito ${ }^{2}, \mathrm{PhD}$; Pedro Figueiredo ${ }^{2,3}, \mathrm{PhD}$; André Seabra ${ }^{2,4}, \mathrm{PhD}$; Romeu Mendes ${ }^{1,2,5}, \mathrm{MD}, \mathrm{PhD}$

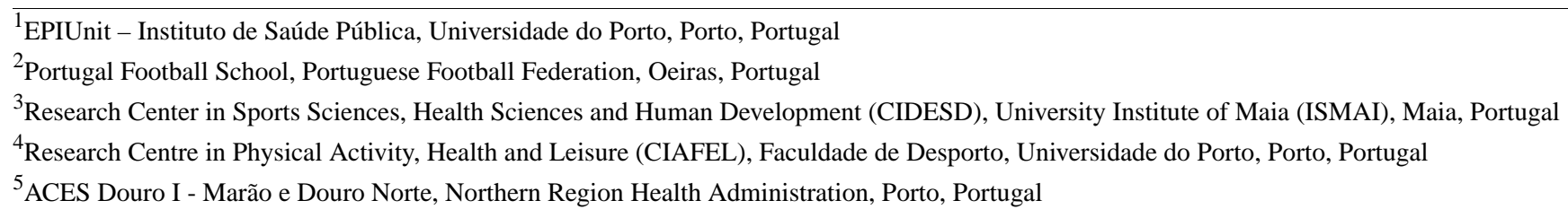

Corresponding Author:

Romeu Mendes, MD, PhD

EPIUnit - Instituto de Saúde Pública

Universidade do Porto

Rua das Taipas, 135

Porto, 4050-600

Portugal

Phone: 351222061820

Email: romeuduartemendes@gmail.com

\section{Abstract}

Background: Studies on walking football have found positive effects on health; however, there are still several research gaps when applying walking football programs for patients with type 2 diabetes.

Objective: This study aims to test the effectiveness of a walking football exercise program on glycemic control and cardiovascular risk factors in middle-aged and older men with type 2 diabetes.

Methods: The study will be run as a randomized controlled trial with a 6-month duration in Portugal. Eligible participants will be randomized using a 1:1 ratio for intervention or control groups and compared using an intention-to-treat analysis. The intervention will consist of a walking football exercise program. The control group will continue with usual care in primary health care units. The primary outcome will be the mean difference in glycated hemoglobin between intervention and control groups after 6 months. Secondary outcomes include the mean differences in fasting blood glucose, total cholesterol, low-density lipoprotein cholesterol, high-density lipoprotein cholesterol, triglycerides, systolic and diastolic blood pressure, body mass index, waist circumference, fat-free mass, and fat mass. Additionally, secondary outcomes include the incidence of exercise-related injuries and adverse events and the walking football exercise program's cost-utility.

Results: The study protocol is being prepared to be submitted to the Health Ethics Committee of the Northern Regional Health Administration, Portugal. After approval, participant recruitment will start in primary health care units in Porto's metropolitan area by family medicine doctors.

Conclusions: Walking football might have the potential to be effective in improving glycemic control and cardiovascular risk factors, with a low rate of exercise-related injuries and adverse events and a good cost-utility ratio. Therefore, walking football may be a sustainable intervention strategy for type 2 diabetes management.

International Registered Report Identifier (IRRID): PRR1-10.2196/28554

(JMIR Res Protoc 2021;10(11):e28554) doi: 10.2196/28554

\section{KEYWORDS}

type 2 diabetes; cardiovascular risk factors; physical activity; exercise; football; soccer; walking; randomized controlled trial 


\section{Introduction}

\section{Background and Rationale}

Type 2 diabetes (T2D) is a global public health concern considering its morbidity, mortality, and health expenditure [1]. In 2019, it was estimated that 463 million adults (20-79 years old) worldwide were diagnosed with diabetes, corresponding to $9.0 \%$ of all adults in this age group [1] and representing nearly $90 \%$ of T2D cases. Increased exposure to environmental factors, such as obesity and physical inactivity, has been associated with the alarming increase in the prevalence of diabetes [1]. Portugal is one of the European countries with the highest prevalence of diabetes. In 2015, the prevalence of diabetes in the adult population (25-74 years old) was $9.9 \%$ [2]. The prevalence was higher in men than in women $(12.1 \%$ vs $7.8 \%)$, and higher in individuals aged $65-74$ years $(23.8 \%)$ compared with younger individuals [2].

The benefits of physical activity in the prevention and control of T2D have long been documented [3-6]; however, a considerable proportion of individuals with T2D do not adhere to the recommendations proposed by international organizations (eg, American Diabetes Association, American College of Sports Medicine) [7,8]. Data from Portugal revealed that about $60 \%$ of individuals with T2D reported not practicing any type of exercise [9], demanding interventions to increase physical activity levels in this population.

Recreational football is conducted as small-sided games, from 3 vs 3 to 7 vs 7, practiced 2-3 times per week, in sessions of 45-60 minutes. The practice of recreational football is an intermittent activity, with participants moving at slow speed, but with consecutive changes in direction, accelerations, and decelerations, leading to periods of moderate-to-vigorous intensity. This intermittent activity has shown cardiovascular, metabolic, and neuromuscular benefits across different populations [10-14]. This can contribute to increased physical activity levels and, therefore, to the control of several noncommunicable diseases, including T2D [10-14].

Exercise-related injuries and the high exercise intensities observed in recreational football led some football clubs to develop walking football strategies for their older players [15]. Walking football follows football's general rules, but it does not allow players to run or have physical contact, and the ball must always be played below the players' average waist height [16]. Available studies on walking football have reported engagement and satisfaction with the modality, the pattern of exercise intensity (from light to vigorous), and health benefits (namely on body composition, aerobic fitness, blood pressure, cognitive function, psychologic well-being, and quality of life) [17-22].

Only 3 studies provided details regarding the participants' medical conditions [17,20,22]. Participants were mainly middle-aged and older men with overweight, obesity, hypertension, or T2D. Characterization of medical conditions and cardiovascular risk factors seems particularly important when extrapolating exercise effects for some populations. Patients with T2D have an increased risk of injuries and acute adverse events associated with exercise training compared with healthy subjects [23]. Indeed, efforts for safety are fundamental in exercise programs and may compromise participants' adherence.

A 12-week study that tested the feasibility and safety of a walking football program in middle-aged and older men with T2D in a quasiexperimental design found that the most common adverse events were falls and musculoskeletal injuries, and no acute metabolic or hemodynamic adverse events were observed. No registered injuries or adverse events were reported, mainly due to the safety protocols applied before, during, and after each exercise session [22].

Studies on walking football showed positive effects on health. However, there are still several research gaps regarding walking football in patients with T2D. It includes limitations in study designs, sample sizes, length of the programs, assessment of variables that may influence adherence to the programs (such as the enjoyment), and impact on glycemic control and cardiovascular risk factors.

\section{Objectives}

This study aims to test the effectiveness of a 6-month walking football exercise program on glycemic control and cardiovascular risk factors in middle-aged and older men with T2D.

This study will be accomplished through the following specific objectives: (1) evaluate the effects of a walking football exercise program on glycemic control, blood lipid profile, blood pressure, anthropometric profile, and body composition; (2) assess exercise-related injuries and adverse events of a walking football exercise program; (3) assess the cost-utility of a walking football exercise program.

\section{Methods}

This protocol follows the Standard Protocol Items: Recommendations for Interventional Trials (SPIRIT) statement [24]. The SPIRIT checklist is available in Multimedia Appendix 1 , and the trial for this protocol will be registered at ClinicalTrials.gov.

\section{Design}

This study is based on a parallel-group, randomized controlled trial with a 6-month duration. Eligible participants will be randomized using a 1:1 ratio within each primary health care unit (PHCU) to intervention or control groups. The intervention will consist of a 60-minute walking football exercise program, 3 times per week (nonconsecutive days), for 24 weeks. The control group will maintain daily life routines and continue with usual care. The study design is represented in Figure 1. 
Figure 1. Flow diagram of the randomized controlled trial.

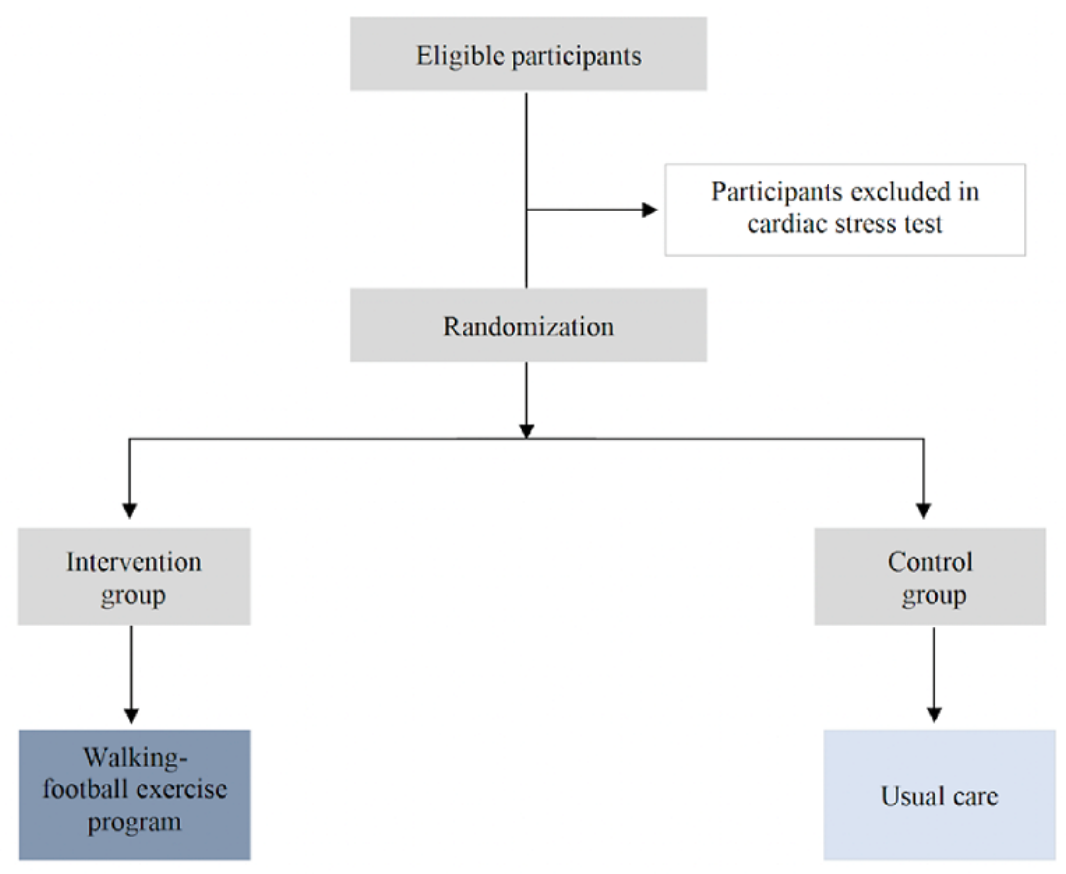

\section{Setting}

The study will be conducted in PHCU of Porto's metropolitan area, in the northern region of Portugal. A PHCU consists of multiprofessional teams, with a mean of 7 family medicine doctors, an equal number of family nurses, and administrative professionals. Family medicine doctors have a patient list that ranges from 1500 to 2000 patients, handling preventive activities and most of the acute and chronic health problems of the individuals. In these units, patients with chronic conditions, such as T2D, have regular consultations and close contact with their family medicine doctor.

\section{Participants}

Participants will be recruited from 5 PHCUs. Family medicine doctors at these PHCUs will extract a list of potential participants from the information system and contact them by telephone. Each PHCU is expected to enroll 40 patients, corresponding to a total of 200 participants.

\section{Inclusion Criteria}

The participants will be selected according to the following criteria: diagnosis of T2D for at least 12 months; male; aged 55-70 years; glycated hemoglobin between $6.0 \%$ and $10.0 \%$; not having started insulin therapy in the previous 6 months and/or sulfonylureas therapy in the previous 3 months; major complications of diabetes screened and controlled (diabetic retinopathy, diabetic nephropathy, and diabetic foot); no cardiovascular, respiratory, nor musculoskeletal contraindications to exercise; without symptoms of coronary artery disease; without limitations in gait or balance; nonsmokers at least for 6 months; not practicing supervised exercise for at least 6 months; independent living in the community; and availability for the exercise session schedule. Participants who

fulfil the inclusion criteria will be invited to participate in the study and perform a treadmill cardiac stress test.

\section{Exclusion Criteria}

Individuals with issues identified in the cardiac stress test, namely asymptomatic cardiac or hemodynamic problems, will be excluded from the study.

\section{Intervention and Control Groups}

Participants assigned to the intervention group will enroll in a walking football exercise program and receive basic sports material (eg, sports bag, t-shirt, and sports shoes). The participants will be organized into 5 groups of 20 players in different time schedules. Each group will have 60-minute walking football exercise sessions, 3 times per week (nonconsecutive days), for 24 weeks (72 sessions).

Walking football sessions will be conducted on a football field and supervised by a football coach certified by the Union of European Football Associations and by a nurse.

The sessions will consist of strength and conditioning exercises, technical skill drills, and small-sided and conditioned walking football games, including warm-up and cool-down periods.

The participants from the intervention and control groups will be asked to maintain daily life routines (lifestyle-related physical activity and dietary pattern) and continue with usual care (diabetes consultations and pharmacological regimen). In Portugal, the usual care at a PHCU already includes brief counseling for physical activity and sedentary behavior [25].

Participants from the control group will also receive basic sports material (eg, sports bag, t-shirt, and sports shoes).

All activities, participation rules, project team members, and the sports facilities will be presented to participants and their families before starting the intervention. 


\section{Outcomes}

The primary outcome is the difference in the change in glycated hemoglobin level between intervention and control groups after 6 months. Secondary outcomes include changes between groups in fasting blood glucose, total cholesterol, low-density lipoprotein cholesterol, high-density lipoprotein cholesterol, triglycerides, systolic and diastolic blood pressure, body mass index, waist circumference, fat-free mass, and fat mass.

Secondary outcomes also include the incidence of exercise-related injuries and adverse events, as well as the cost-utility of the walking football exercise program.

\section{Assignment of Interventions}

The principal investigator will use a computerized random number generator to randomize participants.

Each patient will have a unique patient study number, which will be given immediately by the randomization software (the latest version of Excel Office 365).

Table 1. Variables assessed at baseline and at the end of the study.

\begin{tabular}{|c|c|}
\hline Variable & Method \\
\hline \multicolumn{2}{|l|}{ Glycemic control } \\
\hline Glycated hemoglobin & Venous blood analysis \\
\hline Fasting blood glucose & Venous blood analysis \\
\hline \multicolumn{2}{|l|}{ Blood lipid profile } \\
\hline Total cholesterol & Venous blood analysis \\
\hline LDL $^{\mathrm{a}}$ cholesterol & Venous blood analysis \\
\hline $\mathrm{HDL}^{\mathrm{b}}$ cholesterol & Venous blood analysis \\
\hline Triglycerides & Venous blood analysis \\
\hline \multicolumn{2}{|l|}{ Blood pressure } \\
\hline Systolic blood pressure & Automatic digital sphygmomanometer \\
\hline Diastolic blood pressure & Automatic digital sphygmomanometer \\
\hline \multicolumn{2}{|l|}{ Anthropometric profile } \\
\hline Body mass index & Formula \\
\hline Waist circumference & Anthropometric tape \\
\hline \multicolumn{2}{|l|}{ Body composition } \\
\hline Fat mass & Bioelectrical impedance analysis \\
\hline Fat-free mass & Bioelectrical impedance analysis \\
\hline Habitual physical activity & Global Physical Activity Questionnaire score [26] \\
\hline Dietary intake & 3-day food record, 24-hour dietary recall analysis \\
\hline Health-related quality of life & EQ-5D questionnaire [27] \\
\hline Medication (number, type, and dosage) & Form \\
\hline
\end{tabular}

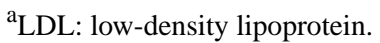

${ }^{\mathrm{b}}$ HDL: high-density lipoprotein.

Before and after the study, habitual physical activity, dietary intake, health-related quality of life, and regular medication will

be collected to be used as control variables. We will also collect sociodemographic characteristics at baseline.
Family medicine doctors at each PHCU will invite patients for an appointment; then, family medicine doctors will provide information about the study, assess the availability for participation, and collect informed written consent. After consent, the participants will be invited to perform a treadmill

If still eligible after the stress test, the participants will be enrolled in the study by the research team, who will provide an opaque envelope with the codification previously generated for each participant; the participant will then open the envelope and check his allocation for the intervention or control group.

Blinding of the participants, health care professionals, and research team members will not be possible.

\section{Data Collection}

Data collection will have 3 main time points: baseline (before starting the study), 6 months (after the study ends), and every walking football exercise session. The descriptions of the variables assessed at baseline and the 6-month evaluation are presented in Table 1. cardiac stress test. 
At every exercise session, objective and subjective exercise intensity and enjoyment will be recorded for control purposes.

The descriptions of the variables to be assessed before, during, and after each exercise session are detailed in Table 2. Capillary blood glucose, blood pressure, and feet wounds will be assessed

Table 2. Variables assessed in each exercise session. before the exercise session; objective exercise intensity will be recorded during the exercise session; subjective exercise intensity and enjoyment will be assessed at the end of the exercise session; and exercise-related injuries and adverse events will be evaluated during and after the exercise session if participants report symptoms or the nurse notices any issue.

\begin{tabular}{ll}
\hline Variable & Method \\
\hline Pre-exercise capillary blood glucose & Glucometer \\
Pre-exercise blood pressure & Automatic digital sphygmomanometer \\
Pre-exercise feet wounds & Self-observation
\end{tabular}

\section{Exercise intensity}

Subjective

Objective

Exercise sessions enjoyment

Exercise-related injuries and adverse events

All personnel involved in the study - nurses, nutritionists, medical doctors, football coaches, and sports scientists - will receive training before baseline assessments. This aims to standardize procedures.

Specifically, the principal investigator will provide training to the other research team members regarding the clinical assessments and forms.

The Portugal Football School of the Portuguese Football Federation will provide training to football coaches to ensure the walking football program is administered in a similar way in the different centers and according to a predefined manual.

A sports scientist will monitor, in real time, internal (heart rate [HR]) and external (eg, distance covered, number of actions) workload and apply the OMNI perceived exertion scale in all sessions.

The nurse must have training in emergency procedures, be responsible for measurements at exercise sessions, manage and record exercise-related injuries and adverse events, follow-up with participants, and refer to health care facilities if necessary.

\section{Procedures}

Before each walking football session, the nurse present at the local sports facility will evaluate capillary blood glucose (Contour XT, Ascencia Diabetes Care, Basel, Switzerland) and blood pressure (M6 Comfort, Omron, Kyoto, Japan) for all participants. Furthermore, participants will self-observe the presence of feet wounds and report any to the nurse. These measurements aim to evaluate the baseline safety conditions before the exercise session.

The participants will be allowed to start the session only under the following conditions: (1) capillary blood glucose $\geq 100$ and $\leq 300 \mathrm{mg} / \mathrm{dL}$, (2) systolic blood pressure $\leq 200 \mathrm{~mm} \mathrm{Hg}$, (3) diastolic blood pressure $\leq 100 \mathrm{~mm} \mathrm{Hg}$, and (4) no foot wounds.

During and after sessions, capillary blood glucose, blood pressure, and feet will be evaluated if participants report related
OMNI perceived exertion scale [28]

Heart rate and time-motion tracking

Physical Activity Enjoyment Scale [29]

Observational/clinical/self-reported

symptoms. An adverse event is considered if there are any of the following after reporting symptoms: capillary blood glucose $<72 \mathrm{mg} / \mathrm{dL}$ (symptomatic hypoglycemia) or $>300 \mathrm{mg} / \mathrm{dL}$ (symptomatic hyperglycemia), systolic blood pressure $<100$ $\mathrm{mm} \mathrm{Hg}$ (symptomatic hypotension) or $>160 \mathrm{~mm} \mathrm{Hg}$ (symptomatic hypertension response), or foot wounds are observed [3,23]. Participants in these conditions will not return to the exercise session, and corrective measures will be applied when necessary (ie, hydration, glucose intake, rest).

Strains, sprains, and contusions will be considered musculoskeletal injuries. Falls, seizures, myalgias, headache, malaise, chest pain and discomfort, and other relevant events will be considered adverse events [22].

During the walking football program, exercise intensity will be monitored systematically in every session through HR and rating of perceived exertion (RPE). From the estimated HR reserve (HRR) [30], we will classify the exercise intensity using the method by Karvonen and Vuorimaa [31]: light intensity (30\%-39\% HRR), moderate intensity (40\%-59\% HRR), vigorous intensity (60\%-89\% HRR), and near-maximal to maximal intensity ( $\geq 90 \%$ HHR) [32]. During training sessions, all participants will use adjustable chest strap HR monitors, and $\mathrm{HR}$ will be recorded at 5-second intervals using short-range radio telemetry (Firstbeat Sports, Jyväskylä, Finland). Participants will classify subjective exercise intensity through RPE using the 11-point OMNI scale (from extremely easy [0 points] to extremely hard [10 points]) at the end of the session [28]. With this RPE scale, light intensity is considered as 3-4 points, moderate intensity as 5-6 points, vigorous intensity as 7-8 points, and near-maximal to maximal intensity as 9-10 points.

\section{Data Analysis and Sample Size}

The number of participants to be involved was defined to test the superiority of walking football compared with usual care, using intention-to-treat analysis. For a 1:1 ratio of the intervention and control groups, significance level of 5\%, and 
statistical power of $80 \%$, a total of 162 participants will be needed to detect a mean difference of at least $0.35 \%$ in the primary outcome (glycated hemoglobin), based on previous meta-analyses $[33,34]$. Assuming the complete follow-up of at least $80 \%$ of the participants, a total of 200 participants will be enrolled.

We will use a 2-way (group*time) analysis of variance with repeated measures to compare the mean differences between intervention and control groups.

For the cost-utility analysis, we will calculate the walking football implementation costs compared with the usual care. Costs include technical training for the professionals involved, patients' medical assessments, material for capillary blood glucose and blood pressure evaluations, football equipment, sports facility rental, sports insurance, and payment to nurses and football coaches. The costs of the intervention and reported gains in quality of life (based on quality-adjusted life years) [27] will be used to calculate the incremental cost-utility ratio (ICUR). ICUR will be compared with the World Health Organization thresholds for health interventions based on per capita gross domestic product.

The amount of missing data is expected to be low considering the training of all the staff and the use of standardized procedures for data collection. No imputation is being planned.

\section{Ethics and Dissemination}

This study will follow the General Data Protection Regulation and be submitted to the Health Ethics Committee of the Northern Regional Health Administration, Portugal. All procedures will comply with the Declaration of Helsinki. Any protocol deviation will be reported.

All participants will provide informed consent after receiving a detailed explanation of the potential risks and benefits. They will also have research insurance to cover the risks associated with exercise practice and evaluations before and after the exercise program. All participants will have a codification number to be used in the evaluations (questionnaires and blood samples). All the data collected will be treated as confidential and strictly used for this project. Data storage will be anonymized. Only the principal investigator will have access to the data.

Participants can withdraw from the study at any time, without any prejudice to the care provided at their PHCU, and have the right to access personal data collected in person from the researchers.

Findings from this study will be submitted for publication in international peer-reviewed journals. The results will also be disseminated at national and international scientific meetings and in mass media press releases.

\section{Results}

The study protocol is being prepared to be submitted to the Health Ethics Committee of the Northern Regional Health Administration, Portugal. After approval, participant recruitment will start by family medical doctors in PHCUs in Porto's metropolitan area.

\section{Discussion}

\section{Overview}

The main goal of this study is to test the effectiveness of a walking football program for glycemic control and cardiovascular risk factors in middle-aged and older male patients with T2D.

To the best of our knowledge, this is the first study testing the effects of a walking football exercise program for these outcomes in this specific population. Also, the investigation proposed relies on robust methodology, with a large study sample.

We expect that the walking football program will be effective in improving diabetes control and cardiovascular risk factors. We also expect a low rate of exercise-related injuries and adverse events and a good cost-utility ratio, as observed in other studies testing the effect of physical activity on diabetes control [35-38].

Developing an effective program with a good cost-utility ratio may contribute to making walking football a sustainable intervention strategy for T2D control. In addition, it can be used by football clubs to offer these programs as a service to their communities, ultimately contributing to the sustainability of the intervention and scaling up the offer to population coverage.

\section{Limitations}

Some limitations need to be addressed: First, the lack of participant, health care professional, and elements of the research team's blinding may introduce bias that may affect the outcome assessment. Nevertheless, our primary outcome is an objective measure - glycated hemoglobin, measured by blood clinical analysis - which may reduce the impact on the lack of blinding.

Second, contamination may occur since randomization units are individuals and not PHCUs, and there is proximity in the geographical area for participants from each PHCU. However, education of all research members and participants towards contamination and clear information about the purposes of the trial may minimize its occurrence.

\section{Acknowledgments}

This work is supported by the Portuguese Foundation for Science and Technology, grant number SFRH/BD/136702/2018. 


\section{Authors' Contributions}

$\mathrm{AB}, \mathrm{RM}$, and JB conceived and designed the study. AB wrote the first version of the manuscript. JB, AS, PF, and RM critically revised the manuscript for relevant intellectual content. All authors approved the final version for submission.

\section{Conflicts of Interest}

None declared.

\section{Multimedia Appendix 1}

The Standard Protocol Items: Recommendations for Interventional Trials checklist. [DOC File, 124 KB-Multimedia Appendix 1]

\section{References}

1. IDF Diabetes Atlas 2019. International Diabetes Federation. URL: https://www.diabetesatlas.org/en/resources/ [accessed 2021-09-26]

2. Barreto M, Kislaya I, Gaio V, Rodrigues AP, Santos AJ, Namorado S, INSEF Research Group. Prevalence, awareness, treatment and control of diabetes in Portugal: Results from the first National Health examination Survey (INSEF 2015). Diabetes Res Clin Pract 2018 Jun;140:271-278. [doi: 10.1016/j.diabres.2018.03.052] [Medline: 29626582]

3. Colberg SR, Sigal RJ, Yardley JE, Riddell MC, Dunstan DW, Dempsey PC, et al. Physical Activity/Exercise and Diabetes: A Position Statement of the American Diabetes Association. Diabetes Care 2016 Nov 25;39(11):2065-2079 [FREE Full text] [doi: 10.2337/dc16-1728] [Medline: 27926890]

4. American Diabetes Association. 5. Facilitating Behavior Change and Well-being to Improve Health Outcomes: Standards of Medical Care in Diabetes-2021. Diabetes Care 2021 Jan;44(Suppl 1):S53-S72. [doi: 10.2337/dc21-S005] [Medline: 33298416]

5. Bull FC, Al-Ansari SS, Biddle S, Borodulin K, Buman MP, Cardon G, et al. World Health Organization 2020 guidelines on physical activity and sedentary behaviour. Br J Sports Med 2020 Dec 25;54(24):1451-1462 [FREE Full text] [doi: 10.1136/bjsports-2020-102955] [Medline: 33239350]

6. Dempsey PC, Friedenreich CM, Leitzmann MF, Buman MP, Lambert E, Willumsen J, et al. Global Public Health Guidelines on Physical Activity and Sedentary Behavior for People Living With Chronic Conditions: A Call to Action. J Phys Act Health 2020 Dec 04;18(1):76-85. [doi: 10.1123/jpah.2020-0525] [Medline: 33276323]

7. Jarvie JL, Pandey A, Ayers CR, McGavock JM, Sénéchal M, Berry JD, et al. Aerobic Fitness and Adherence to Guideline-Recommended Minimum Physical Activity Among Ambulatory Patients With Type 2 Diabetes Mellitus. Diabetes Care 2019 Jul 21;42(7):1333-1339 [FREE Full text] [doi: 10.2337/dc18-2634] [Medline: 31221698]

8. Zhao G, Ford ES, Li C, Balluz LS. Physical activity in U.S. older adults with diabetes mellitus: prevalence and correlates of meeting physical activity recommendations. J Am Geriatr Soc 2011 Jan;59(1):132-137. [doi: 10.1111/j.1532-5415.2010.03236.x] [Medline: 21226683]

9. Mendes R, Dias E, Gama A, Castelo-Branco M, Themudo-Barata JL. Prática de exercício físico e níveis de atividade física habitual em doentes com diabetes tipo 2 - estudo piloto em Portugal. Revista Portuguesa de Endocrinologia, Diabetes e Metabolismo 2013 Jan;8(1):9-15. [doi: 10.1016/j.rpedm.2012.05.001]

10. Milanović Z, Pantelić S, Čović N, Sporiš G, Mohr M, Krustrup P. Broad-spectrum physical fitness benefits of recreational football: a systematic review and meta-analysis. Br J Sports Med 2019 Aug 25;53(15):926-939 [FREE Full text] [doi: 10.1136/bjsports-2017-097885] [Medline: 29371223]

11. Luo H, Newton RU, Ma'ayah F, Galvão DA, Taaffe DR. Recreational soccer as sport medicine for middle-aged and older adults: a systematic review. BMJ Open Sport Exerc Med 2018 Aug 09;4(1):e000336 [FREE Full text] [doi:

10.1136/bmjsem-2017-000336] [Medline: 30112181]

12. Barbosa A, Brito J, Figueiredo P, Seabra A, Mendes R. Football can tackle type 2 diabetes: a systematic review of the health effects of recreational football practice in individuals with prediabetes and type 2 diabetes. Res Sports Med 2021 Jun 22;29(3):303-321. [doi: 10.1080/15438627.2020.1777417] [Medline: 32567951]

13. Schmidt JF, Andersen TR, Horton J, Brix J, Tarnow L, Krustrup P, et al. Soccer training improves cardiac function in men with type 2 diabetes. Med Sci Sports Exerc 2013 Dec;45(12):2223-2233. [doi: 10.1249/MSS.0b013e31829ab43c] [Medline: 23669882]

14. de Sousa MV, Fukui R, Krustrup P, Pereira RMR, Silva PRS, Rodrigues AC, et al. Positive effects of football on fitness, lipid profile, and insulin resistance in Brazilian patients with type 2 diabetes. Scand J Med Sci Sports 2014 Aug 19;24(Suppl 1):57-65. [doi: 10.1111/sms.12258] [Medline: 24944132]

15. Dönmez G, Korkusuz F, Özçakar L, Karanfil Y, Dursun E, Kudaş S, et al. Injuries Among Recreational Football Players: Results of a Prospective Cohort Study. Clin J Sport Med 2018 May;28(3):249-254. [doi: 10.1097/JSM.0000000000000425] [Medline: 28727642]

16. FA revises laws of the game for walking football. The Football Association. 2018. URL: http://www.thefa.com/news/2018/ oct/08/walking-football-revised-laws-of-the-game-081018 [accessed 2021-02-16] 
17. Arnold JT, Bruce-Low S, Sammut L. The impact of 12 weeks walking football on health and fitness in males over 50 years of age. BMJ Open Sport Exerc Med 2015 Oct 08;1(1):bmjsem-2015-000048 [FREE Full text] [doi: 10.1136/bmjsem-2015-000048] [Medline: 27900112]

18. Cholerton R, Breckon J, Butt J, Quirk H. Experiences Influencing Walking Football Initiation in 55- to 75-Year-Old Adults: A Qualitative Study. J Aging Phys Act 2019 Dec 10;10:1-13. [doi: 10.1123/japa.2019-0123] [Medline: 31825889]

19. Harper LD, Field A, Corr LD, Naughton RJ. The Physiological, Physical, and Biomechanical Demands of Walking Football: Implications for Exercise Prescription and Future Research in Older Adults. J Aging Phys Act 2019 Dec 10;10:1-11. [doi: 10.1123/japa.2019-0330] [Medline: $\underline{\text { 31825890] }}$

20. McEwan G, Buchan D, Cowan D, Arthur R, Sanderson M, Macrae E. Recruiting Older Men to Walking Football: A Pilot Feasibility Study. Explore (NY) 2019 May;15(3):206-214. [doi: 10.1016/j.explore.2018.12.001] [Medline: 30639074]

21. Reddy P, Dias I, Holland C, Campbell N, Nagar I, Connolly L, et al. Walking football as sustainable exercise for older adults - A pilot investigation. Eur J Sport Sci 2017 Jun 19;17(5):638-645. [doi: 10.1080/17461391.2017.1298671] [Medline: $28316258]$

22. Barbosa A, Brito J, Costa J, Figueiredo P, Seabra A, Mendes R. Feasibility and safety of a walking football program in middle-aged and older men with type 2 diabetes. Prog Cardiovasc Dis 2020 Nov;63(6):786-791. [doi: 10.1016/j.pcad.2020.06.014] [Medline: 32634461]

23. Mendes R, Sousa N, Reis VM, Themudo-Barata JL. Prevention of exercise-related injuries and adverse events in patients with type 2 diabetes. Postgrad Med J 2013 Dec;89(1058):715-721. [doi: 10.1136/postgradmedj-2013-132222] [Medline: 24194555]

24. Chan A, Tetzlaff JM, Altman DG, Laupacis A, Gøtzsche PC, Krleža-Jerić K, et al. SPIRIT 2013 statement: defining standard protocol items for clinical trials. Ann Intern Med 2013 Feb 05;158(3):200-207 [FREE Full text] [doi: 10.7326/0003-4819-158-3-201302050-00583] [Medline: 23295957]

25. Mendes R, Nunes Silva M, Santos Silva C, Marques A, Godinho C, Tomás R, et al. Physical Activity Promotion Tools in the Portuguese Primary Health Care: An Implementation Research. Int J Environ Res Public Health 2020 Jan 28;17(3):815 [FREE Full text] [doi: 10.3390/ijerph17030815] [Medline: 32012974]

26. Bull F, Maslin TS, Armstrong T. Global physical activity questionnaire (GPAQ): nine country reliability and validity study. J Phys Act Health 2009 Nov;6(6):790-804. [doi: 10.1123/jpah.6.6.790] [Medline: 20101923]

27. Ferreira LN, Ferreira PL, Pereira LN, Oppe M. The valuation of the EQ-5D in Portugal. Qual Life Res 2014 Mar 8;23(2):413-423. [doi: 10.1007/s11136-013-0448-z] [Medline: 23748906]

28. Utter AC, Robertson RJ, Green JM, Suminski RR, McAnulty SR, Nieman DC. Validation of the Adult OMNI Scale of perceived exertion for walking/running exercise. Med Sci Sports Exerc 2004 Oct;36(10):1776-1780. [doi: 10.1249/01.mss.0000142310.97274.94] [Medline: $\underline{15595300]}$

29. Teques P, Calmeiro L, Silva C, Borrego C. Validation and adaptation of the Physical Activity Enjoyment Scale (PACES) in fitness group exercisers. J Sport Health Sci 2020 Jul;9(4):352-357 [FREE Full text] [doi: 10.1016/j.jshs.2017.09.010] [Medline: $\underline{32768128]}$

30. Tanaka H, Monahan KD, Seals DR. Age-predicted maximal heart rate revisited. Journal of the American College of Cardiology 2001 Jan;37(1):153-156. [doi: 10.1016/s0735-1097(00)01054-8]

31. Karvonen J, Vuorimaa T. Heart rate and exercise intensity during sports activities. Practical application. Sports Med 1988 May;5(5):303-311. [doi: 10.2165/00007256-198805050-00002] [Medline: 3387734]

32. Garber CE, Blissmer B, Deschenes MR, Franklin BA, Lamonte MJ, Lee IM, American College of Sports Medicine. American College of Sports Medicine position stand. Quantity and quality of exercise for developing and maintaining cardiorespiratory, musculoskeletal, and neuromotor fitness in apparently healthy adults: guidance for prescribing exercise. Med Sci Sports Exerc 2011 Jul;43(7):1334-1359. [doi: 10.1249/MSS.0b013e318213fefb] [Medline: 21694556]

33. Snowling NJ, Hopkins WG. Effects of different modes of exercise training on glucose control and risk factors for complications in type 2 diabetic patients: a meta-analysis. Diabetes Care 2006 Nov 25;29(11):2518-2527. [doi: 10.2337/dc06-1317] [Medline: 17065697]

34. Boulé NG, Haddad E, Kenny GP, Wells GA, Sigal RJ. Effects of exercise on glycemic control and body mass in type 2 diabetes mellitus: a meta-analysis of controlled clinical trials. JAMA 2001 Sep 12;286(10):1218-1227. [doi: 10.1001/jama.286.10.1218] [Medline: 11559268]

35. Kumar AS, Maiya AG, Shastry BA, Vaishali K, Ravishankar N, Hazari A, et al. Exercise and insulin resistance in type 2 diabetes mellitus: A systematic review and meta-analysis. Ann Phys Rehabil Med 2019 Mar;62(2):98-103 [FREE Full text] [doi: 10.1016/j.rehab.2018.11.001] [Medline: 30553010]

36. Munan M, Oliveira CLP, Marcotte-Chénard A, Rees JL, Prado CM, Riesco E, et al. Acute and Chronic Effects of Exercise on Continuous Glucose Monitoring Outcomes in Type 2 Diabetes: A Meta-Analysis. Front Endocrinol (Lausanne) 2020 Aug 4;11:495 [FREE Full text] [doi: 10.3389/fendo.2020.00495] [Medline: 32849285]

37. Leal J, Ahrabian D, Davies MJ, Gray LJ, Khunti K, Yates T, et al. Cost-effectiveness of a pragmatic structured education intervention for the prevention of type 2 diabetes: economic evaluation of data from the Let's Prevent Diabetes cluster-randomised controlled trial. BMJ Open 2017 Jan 09;7(1):e013592 [FREE Full text] [doi: 10.1136/bmjopen-2016-013592] [Medline: 28069625] 
38. Coyle D, Coyle K, Kenny GP, Boulé NG, Wells GA, Fortier M, et al. Cost-Effectiveness of Exercise Programs in Type 2 Diabetes. Int J Technol Assess Health Care 2012 Jul;28(3):228-234. [doi: 10.1017/s0266462312000256]

\section{Abbreviations}

HR: heart rate

HRR: heart rate reserve

ICUR: incremental cost-utility ratio

PHCU: primary health care unit

RPE: rating of perceived exertion

SPIRIT: Standard Protocol Items: Recommendations for Interventional Trials

T2D: type 2 diabetes

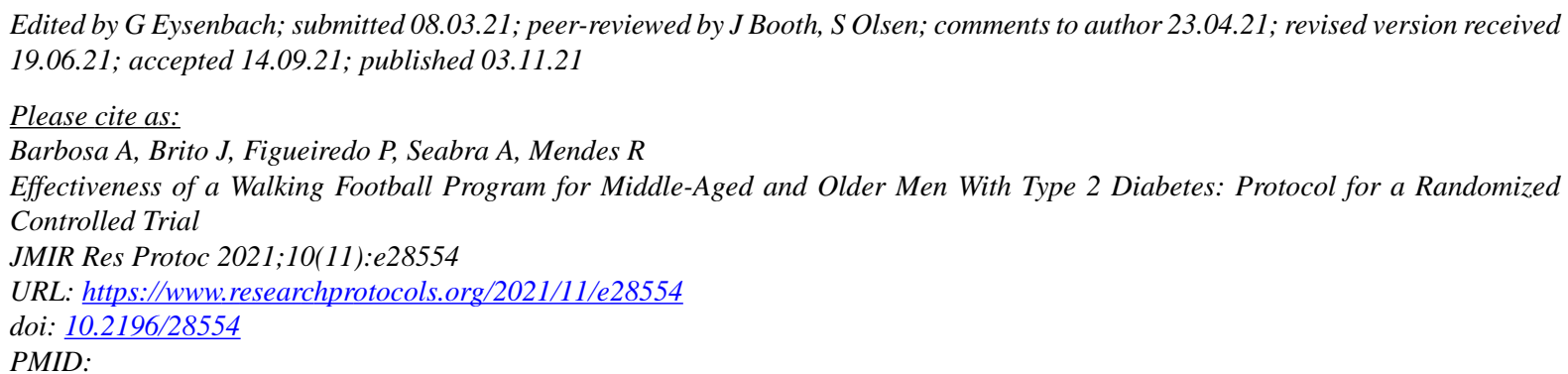

CCAna Barbosa, João Brito, Pedro Figueiredo, André Seabra, Romeu Mendes. Originally published in JMIR Research Protocols (https://www.researchprotocols.org), 03.11.2021. This is an open-access article distributed under the terms of the Creative Commons Attribution License (https://creativecommons.org/licenses/by/4.0/), which permits unrestricted use, distribution, and reproduction in any medium, provided the original work, first published in JMIR Research Protocols, is properly cited. The complete bibliographic information, a link to the original publication on https://www.researchprotocols.org, as well as this copyright and license information must be included. 